\title{
Recent progress in Bistatic SAR with illuminators of opportunity
}

\author{
ZENG Tao, ZHANG Tian, TIAN WeiMing** \& HU Cheng \\ Beijing Key Laboratory of Embedded Real-time Information Processing Technology, School of Information and Electronics, \\ Beijing Institute of Technology, Beijing 100081, China
}

Received October 8, 2016; accepted October 24, 2016; published online November 18, 2016

Bistatic SAR (BiSAR) with illuminators of opportunity is a kind of flexible SAR system that consists of non-cooperative transmitters and a stationary or moving receiver, as Figure 1 shows. In recent years, notable efforts have been made to promote the development of this kind of system. Progress regarding BiSAR with navigation satellites as transmitters before 2015 , including the related theory and practice, has been well reviewed by Antoniou et al. and can be referred from [1]. This paper reviews some of the progress in 2015 and 2016, including novel signal processing approaches, advanced BiSAR system configurations and potentially valuable applications such as distributed scatterers profile extraction, local area surface change detection, and Digital Elevation Model (DEM) generation, etc.

As Global Navigation Satellite System (GNSS) consists of a large number of satellites with various orbit parameters, ideas of utilizing this feature have been conceived. Zeng et al. [2] proposed a multi-angle BiSAR imaging technique. In their work, BeiDou-2/Compass-2 navigation satellites with different observing angles were selected as the illuminators and the corresponding BiSAR images were obtained and fused to be one image. Image difference because of target scattering characteristic under various angles was observed in the experimental data and a Bayesian method was proposed to combine the images, which can extract some significant features from images in specific angles and can also obtain different parts of one distributed scatterers, yielding images with continuous profiles as well as featured details.

Inspired by the fact that various geometry leads to different range and azimuth resolution directions, Santi et al. [3] proposed a spatial resolution improvement technique based on multi-static acquisition and feature extraction. BiSAR images under different angles were obtained and

\footnotetext{
*Corresponding author (email: tianwei6779@163.com)
}

incoherently fused into one image. As the side-lobes of two points targets could generate "ghost" targets in the intersection positions, the combined image could not provide accurate information of the illuminated region. To resolve this issue, they proposed an ad hoc CLEAN-like technique. This technique features on an iterative processing step, where the position and intensity of the brightest target in every iterative step were estimated based on the point spread function (PSF) under the specific geometry and then the point could be removed along with its side-lobes. As the "ghost" target only appears when more than one related targets profile exists, the aforementioned iterative estimating and removing processing can ensure free of "ghost" target. Finally, the image with point targets could be obtained based on the recorded positions of the point targets.

Due to the spatial separation of the transmitter and receiver, Global Navigation Satellite System-based (GNSSbased) BiSAR confronts with time and frequency synchronization problems, which can result in defocusing of BiSAR images. In ref. [4], the authors proposed an integrative synchronization and imaging method to tackle the problems. The effects of the navigation signal, which exhibited as irregular phase jumps along the synthetic aperture time, were firstly estimated and removed to implement pre-synchronization processing. After that, the principle of error neutralization was successfully applied to cancel the time and frequency errors of echo signal. Finally, the traditional back-projection algorithm could be utilized to obtain the focused images.

Some significant efforts have been made to improve the BiSAR image quality in both theoretical and practical levels. Ma et al. [5] put forward a signal bandwidth combination scheme to improve the range resolution of BiSAR images. In their research, the Galileo satellite was selected as the illuminator and its E5a and E5b signals, which were near in 


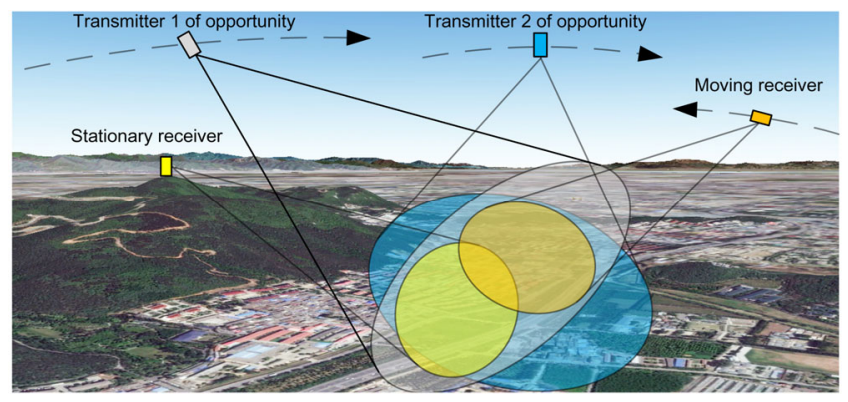

Figure 1 (Color online) Illustration of Bistatic SAR with illuminators of opportunity.

frequency, were collected simultaneously. To implement bandwidth synthesis, a compensation factor was obtained through power spectral density estimation of the theoretical combined signal, which was then used to compensate the coherently-added two channels signals to form one wider bandwidth signal. In this way, 5 times of range resolution improvement could be achieved. Wang et al. [6] proposed a digital beamforming (DBF) scheme with TerraSAR-X as the illuminator. In their BiSAR system, 5 echo antennas were emplaced vertically with a certain distance and multi-channel receiver was deployed to receive the signals from the direct signal antenna and echo antennas. DBF method was applied which could yield a sharper and higher antenna pattern. In this way, images with higher SNR could be obtained.

In the last two years, some novel applications of bistatic SAR has been investigated. One is about the surface change detection, which has been researched by Zeng et al. [7]. In their work, a subsidence monitoring technique based on bistatic differential interferometry using GNSS as illuminators was presented. A transponder was placed on the region of interest to enable strong reflection. The principles of Differential GNSS (DGNSS) and the SAR technique were combined to eliminate some common errors like the unknown ionosphere and tropospheric delays, as well as to increase the signal SNR through long time integration, which could remarkably improve the subsidence estimation accuracy. A field experiment was carried out to validate its millimeter accuracy ability of deformation measurement. Another application is regarding the DEM generation through Space-Surface BiSAR interferometry with noncooperative SAR radar satellites as illuminators, which has been researched by both Zeng et al. [8] and Hong et al. [9]. In ref. [8], the YaoGan-3 (an L-band space-borne SAR) was selected as the transmitter and the two stationary receivers were mounted on the top of a tall building. An iterative LS approach was proposed to tackle the problem of inaccurate antennas positions in the receiver end, successfully achieving DEM generation with an accuracy of about 1-2 $\mathrm{m}$ for some targets. In ref. [9], a multi-channel system was built to enable solving problems of DEM generation in regions comprising buildings, where the discontinuity effect occur- ring in the edges of buildings in the interferogram was severe. A multi-channel DEM generation method based on Markov Random Field (MRF) was proposed, where the amplitude data, iterative reconstruction results, and Maximum Likelihood (ML) results were jointly utilized to optimize the total MRF energy function. TerraSAR-X was selected as illuminators and the field experiment has been conducted to validate the method. A DEM generation accuracy of $0.98 \mathrm{~m}$ was achieved in the experiment.

Apart from GNSS and SAR radar satellites, Gromek et al. [10] conducted a pioneering experiment using a Commercial Digital Video Broadcasting-Terrestrial (DVB-T) station as a stationary transmitter, and an airborne passive radar as a receiver. The advantage of DVB-T is its high transmitting power (with EIRP about $100 \mathrm{~kW}$ ), making it unnecessary to integrate long synthetic aperture time to achieve sufficient SNR gain like GNSS-based BiSAR does. The experiment result shows that the system can obtain the terrain profile, indicating its huge potential in local area monitoring.

Although the very rapid development of BiSAR with illuminators of opportunity has been witnessed in recent years, this kind of system is still far from maturity. Considering some of the existing shortages and the system peculiarities, the future works and development trends may include:

(1) In the theoretical level, the scattering characteristics of illuminated targets in BiSAR mode is worth researching on. Due to the special geometry, BiSAR images exhibit some different features from their monostatic counterparts. Many BiSAR images have been obtained but the interpretation is not sufficient yet, especially for the interpretation of the GNSS-based BiSAR images, which is very challenging due to the poor image resolution. The key techniques may comprise electromagnet modelling of complex object under low band signal and multiple times signal reflection.

(2) In the technical level, approaches to improve the image quality could be further researched. Parameterized imaging method, which considers the actual object model instead of using the traditional point scattering model may help improve the imaging result with limited image resolution. Besides, wide signal band synthesis and very long synthetic aperture time imaging are promising to improve the image resolution. The key techniques may include synthesis error compensation and efficient imaging algorithm with huge raw data.

(3) In the application level, surface change detection in real scenarios could be investigated, especially for the GNSS satellites which have short revisit cycle and abundant number. The key techniques may be atmosphere error removal, high accurate DEM generation, and receiver side error compensation.

This work was supported by the National Natural Science Foundation of China (Grant Nos. 61120106004, 61427802, 61225005, 61601031), Chang 
Jiang Scholars Program (Grant No. T2012122), and 111 project of China (Grant No. B14010).

1 Antoniou M, Cherniakov M, Ma H. Space-surface bistatic synthetic aperture radar with navigation satellite transmissions: A review. Sci China Inf Sci, 2015, 58: 061301

2 Zeng T, Ao D, Hu C, et al. Multiangle BSAR imaging based on BeiDou-2 navigation satellite system: Experiments and preliminary results. IEEE Trans Geosci Remote Sensing, 2015, 53: 5760-5773

3 Santi F, Bucciarelli M, Pastina D, et al. Spatial resolution improvement in GNSS-Based SAR using multistatic acquisitions and feature extraction. IEEE Trans Geosci Remote Sensing, 2016, 54: 6217-6231

4 Zhang Q, Chang W, Zeng Z, et al. An integrative synchronization and imaging algorithm for GNSS-based BSAR. Sci China Inf Sci, 2015, 58: 062303

5 Ma H, Antoniou M, Cherniakov M. Passive GNSS-based SAR imaging and opportunities using Galileo E5 signals. Sci China Inf Sci, 2015, 58: 062305

6 Wang R, Wang W, Shao Y, et al. First bistatic demonstration of digital beamforming in elevation with TerraSAR-X as an illuminator. IEEE Trans Geosci Remote Sensing, 2016, 54: 842-849

7 Zeng $\mathrm{T}$, Zhang $\mathrm{T}$, Tian $\mathrm{W}$, et al. A novel subsidence monitoring technique based on space-surface bistatic differential interferometry using GNSS as transmitters. Sci China Inf Sci, 2015, 58: 062304

8 Zeng T, Zhu M, Hu C, et al. DEM generation using bistatic interferometry: High-coherence pixel selection and residual reference phase compensation. Sci China Inf Sci, 2015, 58: 062301

9 Hong F, Tang J, Lu P. Multichannel DEM reconstruction method based on Markov random fields for bistatic SAR. Sci China Inf Sci, 2015, 58: 062302

10 Gromek D, Kulpa K, Samczyński P. Experimental results of passive SAR imaging using DVB-T illuminators of opportunity. IEEE Geosci Remote Sensing Lett, 2016, 13: 1124-1128 